\title{
Optical solitons and conservation laws associated with Kudryashov's sextic power-law nonlinearity of refractive index
}

${ }^{1}$ Elsayed M. E. Zayed, ${ }^{1}$ Reham M. A. Shohib, ${ }^{1}$ Mohamed E. M. Alngar, ${ }^{2,3,4,5}$ Anjan Biswas, ${ }^{6}$ Mehmet Ekici, ${ }^{2}$ Salam Khan, ${ }^{3}$ Abdullah Khamis Alzahrani and ${ }^{7}$ Milivoj R. Belic

${ }^{1}$ Mathematics Department, Faculty of Science, Zagazig University, Zagazig 44519, Egypt

${ }^{2}$ Department of Physics, Chemistry and Mathematics, Alabama A\&M University, Normal, AL 35762-4900, USA

${ }^{3}$ Mathematical Modeling and Applied Computation (MMAC) Research Group, Department of Mathematics, King Abdulaziz University, Jeddah 21589, Saudi Arabia

${ }^{4}$ Department of Applied Mathematics, National Research Nuclear University, 31 Kashirskoe Highway, Moscow 115409, Russian Federation

${ }^{5}$ Department of Mathematics and Applied Mathematics, Sefako Makgatho Health Sciences University, Medunsa 0204, Pretoria, South Africa

${ }^{6}$ Department of Mathematics, Faculty of Science and Arts, Yozgat Bozok University, 66100 Yozgat, Turkey

${ }^{7}$ Science Program, Texas A\&M University at Qatar, PO Box 23874, Doha, Qatar

Received: 10.10 .2020

\begin{abstract}
We recover the cases of solutions in the shape of bright, dark and singular optical solitons for the self-phase modulation effect, which belongs to the type of N. A. Kudryashov's sextic power-law nonlinearity of refractive index. Three different integration schemes have been implemented. These are a unified Riccati equation, our new mapping scheme and our addendum to the common N. A. Kudryashov's method. All of the solitons are enlisted and the criterions of their existence are mentioned. Finally, we extract three appropriate conservation laws.
\end{abstract}

Keywords: refractive index, sextic -power nonlinearities, Kudryashov's method, solitons.

UDC: 535.32

\section{Introduction}

Nowadays we witness an avalanche of studies on various nonlinearities of refractive index in telecommunications. Kerr nonlinearity represents the most common form of self-phase modulation, when the refractive index is proportional to the intensity of light. In more general 'non-Kerr' cases, it is proportional to some function of the intensity [1-5]. In other words, the refractive index in the case of Kerr nonlinearity is a constant multiple of the intensity, while in a non-Kerr situation, the response of optical medium sometimes depends on the intensity raised to a positive power or, more generally, it is a sum of two terms, each of which being proportional to the intensity raised to some positive power. Still another form of non-Kerr nonlinearity happens when the refractive index is proportional to logarithm of the intensity, which leads to Gausson solutions, as opposed to more common soliton solutions.

Recently, N. A. Kudryashov has suggested a number of forms for the self-phase modulation effect, which have sparked a great interest among physicists and telecommunication engineers 
[6-16]. The present work addresses soliton solutions of the governing nonlinear Schrödinger's equation, which come from one of the latest forms of refractive-index nonlinearity introduced by N. A. Kudryashov [13]. This nonlinear dependence of the refractive index involves six terms, each of them including a power-law component. This recent nonlinearity of the refractive index still lacks its further theoretical analysis. Moreover, this nonlinear form of the refractive index still awaits the studies answering which of materials can be described by it.

We employ three integration algorithms which, when being applied to the governing nonlinear Schrödinger's equation, can reveal soliton solutions in the model. These are a unified Riccati equation, a new mapping method and an addendum to a known Kudryashov's scheme. It turns out that all of these algorithms are able to yield in so-called 'bright', 'dark' and singular soliton solutions. Our subsequent aim is to determine relevant conservation laws for the governing model. These results accomplish our analysis of the new nonlinearity model. The details to be sketched before the analysis are as follows.

The governing nonlinear Schrödinger's equation with the Kudryashov's sextic power law for the refractive index can be written as [13]

$$
i q_{t}+a q_{x x}+\left(b_{1}|q|^{n}+b_{2}|q|^{2 n}+b_{3}|q|^{3 n}+b_{4}|q|^{4 n}+b_{5}|q|^{5 n}+b_{6}|q|^{6 n}\right) q=0
$$

where $a$ and $b_{j},(j=1,2, \ldots, 6)$ are real-valued constants, while $x$ and $t$ denote the indices of the variables $q(x, t)$. In particular, $q_{t}$ and $q_{x x}$ represent respectively the first- and second-order partial derivatives of $q(x, t)$ with respect to the variables $t$ and $x$. The dependent variable $q(x, t)$ is complex-valued and describes the optical pulse profile. Here $a$ represents the coefficient of chromatic dispersion, $b_{j}$ are the power-law nonlinearity parameters accounting for the self-phase modulation, and $n$ refers to the type of power-law nonlinearity. Finally, the first term in Eq. (1) accounts for the linear temporal evolution.

The rest of the article is organized as follows. We elaborate mathematical analysis in Section 2. In Sections 3, 4 and 5, our algorithms reveal and describe quantitatively the bright, dark and singular optical soliton solutions of Eq. (1). The corresponding conservation laws are enlisted in Section 6. Finally, some conclusive observations are made in Section 7.

\section{Mathematical preliminaries}

We assume that Eq. (1) has a solution in the form

$$
q(x, t)=\varphi(\xi) e^{i(-k x+\omega t+\theta)}, \xi=x-v t,
$$

where $v, \omega, k$ and $\theta$ are nonzero constants to be determined. In particular, the parameter $v$ represents the soliton velocity. From the phase components, $k$ defines the wave number of the soliton, while $\omega$ is the frequency and $\theta$ the phase constant. Here $\varphi(\xi)$ represents a real-valued function that stands for the pulse shape.

Inserting Eq. (2) into Eq. (1) and separating real and imaginary parts, one has

$$
a \varphi^{\prime \prime}-\left(\omega+a k^{2}\right) \varphi+b_{1} \varphi^{1+n}+b_{2} \varphi^{1+2 n}+b_{3} \varphi^{1+3 n}+b_{4} \varphi^{1+4 n}+b_{5} \varphi^{1+5 n}+b_{6} \varphi^{1+6 n}=0
$$

and

$$
v=-2 a k,
$$

where $\varphi^{\prime \prime}$ denotes the second-order derivative with respect to its variable and the remaining derivatives involve exponents. Hence, Eq. (4) gives the velocity of the soliton. Setting

$$
\varphi(\xi)=H^{\frac{1}{3 n}}(\xi),
$$

with $H(\xi)$ being a new positive function of $\xi$, we get the equation 


$$
\begin{aligned}
& a\left[3 n H H^{\prime \prime}+(1-3 n) H^{\prime 2}\right]-9 n^{2}\left(\omega+a k^{2}\right) H^{2}+9 n^{2} b_{1} H^{\frac{7}{3}}+9 n^{2} b_{2} H^{\frac{8}{3}} \\
& +9 n^{2} b_{3} H^{3}+9 n^{2} b_{4} H^{\frac{10}{3}}+9 n^{2} b_{5} H^{\frac{11}{3}}+9 n^{2} b_{6} H^{4}=0
\end{aligned}
$$

For the purposes of integrability, one must select

$$
b_{1}=b_{2}=b_{4}=b_{5}=0 \text {. }
$$

Consequently, Eq. (6) can be modified to

$$
a\left[3 n H H^{\prime \prime}+(1-3 n) H^{\prime 2}\right]-9 n^{2}\left(\omega+a k^{2}\right) H^{2}+9 n^{2} b_{3} H^{3}+9 n^{2} b_{6} H^{4}=0 .
$$

Balancing $H H^{\prime \prime}$ with $H^{4}$ in Eq. (8) yields in the balance number $N=1 \quad(N=1 \div n)$. This concept stems from the balancing principle for the existence of solitons. It states that a soliton is outcome of a delicate balance between dispersion and nonlinearity. Thus, this balance should refer to the highest-order dispersion and the highest-order of nonlinearity.

Now the main problem is to solve Eq. (8), using the three integration schemes mentioned above, i.e. the unified Riccati equation method, our new mapping scheme and our addendum to the common Kudryashov's algorithm. These integration mechanisms will be implemented in the next three sections to retrieve the soliton solutions of the governing model.

\section{Unified Riccati equation}

According to this method, we assume that Eq. (8) has the formal solution

$$
H(\xi)=\alpha_{0}+\alpha_{1} F(\xi)
$$

where $\alpha_{0}$ and $\alpha_{1}$ are constants to be determined, such that $\alpha_{1} \neq 0$. The function $F(\xi)$ satisfies the Riccati equation

$$
F^{\prime}(\xi)=C_{0}+C_{1} F(\xi)+C_{2} F^{2}(\xi),
$$

where $C_{j}(j=0,1,2)$ are constants $\left(C_{2} \neq 0\right)$. It is well known (see, e.g., Ref. [17]) that Eq. (10) has the following fractional solutions:

$$
\begin{gathered}
F(\xi)=-\frac{C_{1}}{2 C_{2}}-\frac{\sqrt{\Delta}}{2 C_{2}}\left[\frac{r_{1} \tanh \left(\frac{\sqrt{\Delta}}{2} \xi\right)+r_{2}}{r_{1}+r_{2} \tanh \left(\frac{\sqrt{\Delta}}{2} \xi\right)}\right], \Delta>0,\left(r_{1}^{2}+r_{2}^{2}\right) \neq 0, \\
F(\xi)=-\frac{C_{1}}{2 C_{2}}+\frac{\sqrt{-\Delta}}{2 C_{2}}\left[\frac{r_{3} \tan \left(\frac{\sqrt{-\Delta}}{2} \xi\right)-r_{4}}{r_{3}+r_{4} \tan \left(\frac{\sqrt{-\Delta}}{2} \xi\right)}\right], \Delta<0,\left(r_{3}^{2}+r_{4}^{2}\right) \neq 0, \\
F(\xi)=-\frac{C_{1}}{2 C_{2}}-\frac{1}{C_{2} \xi+\zeta_{0}}, \Delta=0,
\end{gathered}
$$

where $r_{e}(e=1,2,3,4)$ are arbitrary constants and $\zeta_{0}$ implies an integration constant, with $\Delta=\left(C_{1}^{2}-4 C_{0} C_{2}\right)$. Substituting Eqs. (9) and (10) into Eq. (8), collecting all the coefficients near 
$F^{l}(\xi), \quad(l=0,1,2,3,4)$ and equalling them to zero, we have the following set of algebraic equations:

$$
\begin{aligned}
& F^{4}(\xi): \alpha_{1}^{2}\left[9 n^{2} \alpha_{1}^{2} b_{6}+a C_{2}^{2}(3 n+1)\right]=0, \\
& F^{3}(\xi): \alpha_{1}\left[36 n^{2} \alpha_{0} \alpha_{1}^{2} b_{6}+6 a n \alpha_{0} C_{2}^{2}+3 a n \alpha_{1} C_{1} C_{2}+9 n^{2} \alpha_{1}^{2} b_{3}+2 a \alpha_{1} C_{1} C_{2}\right]=0, \\
& F^{2}(\xi): \alpha_{1}\left[-54 n^{2} \alpha_{0}^{2} \alpha_{1} b_{6}-9 a n \alpha_{0} C_{1} C_{2}-27 n^{2} \alpha_{0} \alpha_{1} b_{3}+9 n^{2} \alpha_{1}\left(\omega+a k^{2}\right)-2 a \alpha_{1} C_{0} C_{2}-a \alpha_{1} C_{1}^{2}\right]=0, \\
& F(\xi): \alpha_{1}\left[-36 n^{2} \alpha_{0}^{3} b_{6}-6 a n \alpha_{0} C_{0} C_{2}-3 a n \alpha_{0} C_{1}^{2}+3 a n \alpha_{1} C_{0} C_{1}-27 n^{2} \alpha_{0}^{2} b_{3}\right] \\
& \quad+\alpha_{1}\left[18 n^{2} \alpha_{0}\left(\omega+a \kappa^{2}\right)-2 a \alpha_{1} C_{0} C_{1}\right]=0, \\
& F^{0}(\xi): 9 n^{2} \alpha_{0}^{4} b_{6}+3 a n \alpha_{0} \alpha_{1} C_{0} C_{1}-3 a n \alpha_{1}^{2} C_{0}^{2}+9 n^{2} \alpha_{0}^{3} b_{3}-9 n^{2} \alpha_{0}^{2}\left(\omega+a k^{2}\right)+a \alpha_{1}^{2} C_{0}^{2}=0 .
\end{aligned}
$$

On solving the above equations with the aid of Maple, one can obtain

$$
\begin{aligned}
& n=n, \omega=\frac{a\left(C_{1}+C_{2}\right)^{2}}{9 n^{2}}-a k^{2}, \alpha_{0}=0, \alpha_{1}= \pm C_{2} \sqrt{-\frac{a(3 n+1)}{9 n^{2} b_{6}}}, \\
& C_{0}=-\frac{\left(C_{2}+2 C_{1}\right)}{4}, b_{3}=-\frac{(3 n+2)\left(C_{1}+C_{2}\right)}{9} \sqrt{-\frac{9 b_{6}}{a n^{2}(3 n+1)}},
\end{aligned}
$$

provided that $a b_{6}<0$. Since $\Delta=\left(C_{1}+C_{2}\right)^{2}>0$, Eq. (1) has the soliton solution

$$
q(x, t)=\left\{ \pm \sqrt{-\frac{a(3 n+1)}{36 n^{2} b_{6}}}\left[C_{1}+\frac{\left(C_{1}+C_{2}\right)\left(r_{1} \tanh \left[\frac{1}{2}\left(C_{1}+C_{2}\right)(x+2 a k t)\right]+r_{2}\right)}{r_{1}+r_{2} \tanh \left[\frac{1}{2}\left(C_{1}+C_{2}\right)(x+2 a k t)\right]}\right]\right\}^{\frac{1}{3 n}} e^{i(-k x+\omega t+\theta)}
$$

In particular, if the conditions $r_{1} \neq 0$ and $r_{2}=0$ hold true in Eq. (16), we deduce that Eq. (1) has the dark soliton solutions:

$$
q(x, t)=\left\{ \pm \sqrt{-\frac{a(3 n+1)}{36 n^{2} b_{6}}}\left[C_{1}+\left(C_{1}+C_{2}\right) \tanh \left(\frac{\left(C_{1}+C_{2}\right)(x+2 a k t)}{2}\right)\right]\right\}^{\frac{1}{3 n}} e^{i(-k x+\omega t+\theta)}
$$

Alternatively, if we set $r_{1}=0$ and $r_{2} \neq 0$ in Eq. (16), then Eq. (1) acquires the singular soliton solutions:

$$
q(x, t)=\left\{ \pm \sqrt{-\frac{a(3 n+1)}{36 n^{2} b_{6}}}\left[C_{1}+\left(C_{1}+C_{2}\right) \operatorname{coth}\left(\frac{\left(C_{1}+C_{2}\right)(x+2 a k t)}{2}\right)\right]\right\}^{\frac{1}{3 n}} e^{i(-k x+\omega t+\theta)}
$$

\section{Mapping scheme}

In the frame of our new mapping method, we adopt that Eq. (8) has the following formal solution:

$$
H(\xi)=A_{0}+A_{1} F(\xi)+A_{2} F^{2}(\xi),
$$

where $A_{0}, A_{1}$ and $A_{2}$ are constants to be determined, such that $A_{2} \neq 0$, and the function $F(\xi)$ satisfies the following first-order equation:

$$
F^{2}(\xi)=r+p F^{2}(\xi)+\frac{q_{1}}{2} F^{4}(\xi)+\frac{s}{3} F^{6}(\xi) .
$$


Here $r, p, q_{1}, s$ are constants ( $\left.s \neq 0\right)$. Substituting Eqs. (19) and (20) into Eq. (8), collecting the coefficients near each power $F(\xi)^{e}\left(F^{\prime}(\xi)\right)^{j},(e=0,1,2, \ldots, 8, j=0,1)$ and setting these coefficients to zero, we arrive at the algebraic equations

$$
\begin{aligned}
& F^{8}(\xi): \frac{1}{3} A_{2}^{2}\left[27 n^{2} A_{2}^{2} b_{6}+4 a s(3 n+1)\right]=0, \\
& F^{7}(\xi): \frac{1}{3} A_{1} A_{2}\left[108 n^{2} A_{2}^{2} b_{6}+a s(21 n+4)\right]=0, \\
& F^{6}(\xi): 36 n^{2} A_{2}^{3}\left(A_{0} b_{6}+\frac{1}{4} b_{3}\right)+\frac{1}{3} A_{2}^{2}\left[162 n^{2} A_{1}^{2} b_{6}+3 a q_{1}(3 n+2)\right]+8 a n A_{0} A_{2} s \\
& +2 a s A_{1}^{2}\left(n+\frac{1}{6}\right)=0, \\
& F^{5}(\xi): A_{1}\left[36 n^{2} A_{2} b_{6}\left(A_{1}^{2}+3 A_{0} A_{2}\right)+27 n^{2} A_{2}^{2} b_{3}+3 a n s A_{0}+2 a q_{1} A_{2}(3 n+1)\right]=0, \\
& F^{4}(\xi): \frac{1}{2} a A_{1}^{2} q_{1}(3 n+1)+4 a A_{2}^{2} p+9 a n A_{0} A_{2} q_{1}-9 n^{2}\left(\omega+a \kappa^{2}\right) A_{2}^{2} \\
& +27 n^{2} A_{2} b_{3}\left(A_{1}^{2}+A_{0} A_{2}\right)+9 n^{2} b_{6}\left(6 A_{0}^{2} A_{2}^{2}+12 A_{0} A_{1}^{2} A_{2}+A_{1}^{4}\right)=0, \\
& F^{3}(\xi):-A_{1}\left[-108 n^{2} A_{0}^{2} A_{2} b_{6}-36 n^{2} A_{0} A_{1}^{2} b_{6}-54 n^{2} A_{0} A_{2} b_{3}-9 n^{2} A_{1}^{2} b_{3}+18\left(\omega+a k^{2}\right) n^{2} A_{2}\right] \\
& +A_{1}\left[3 a p A_{2}(3 n+4)+3 a n q_{1} A_{0}\right]=0, \\
& F^{2}(\xi): 36 n^{2} A_{0}^{3} A_{2} b_{6}+54 n^{2} A_{0}^{2} A_{1}^{2} b_{6}+27 n^{2} A_{0} b_{3}\left(A_{0} A_{2}+A_{1}^{2}\right)-9 n^{2}\left(\omega+a k^{2}\right)\left(A_{1}^{2}+2 A_{0} A_{2}\right) \\
& +12 a n p A_{0} A_{2}-6 a n r A_{2}^{2}+a p A_{1}^{2}+4 a r A_{2}^{2}=0, \\
& F(\xi):-A_{1}\left[-36 n^{2} A_{0}^{3} b_{6}-27 n^{2} A_{0}^{2} b_{3}+18 A_{0} n^{2}\left(\omega+a k^{2}\right)-3 a n p A_{0}+6 a n r A_{2}-4 a r A_{2}\right]=0, \\
& F^{0}(\xi): a(1-3 n) A_{1}^{2} r+6 a n r A_{0} A_{2}-9 n^{2} A_{0}^{2}\left(\omega+a k^{2}\right)+9 n^{2} A_{0}^{3} b_{3}+9 n^{2} A_{0}^{4} b_{6}=0 .
\end{aligned}
$$

With reference to the work [18], one can distinguish three types of solutions of the above algebraic equations, which are outlined below.

Type 1. Substituting $s=\frac{3 q_{1}^{2}}{16 p}$ and $r=\frac{16 p^{2}}{27 q_{1}}$ into Eqs. (21) and solving them with Maple or Mathematica, we have the following results:

$$
\begin{aligned}
& n=n, \quad \omega=\frac{3(3 n+1) b_{3}^{2}}{(3 n+2)^{2} b_{6}}-a k^{2}, \\
& A_{0}=\frac{(3 n+1) b_{3}}{(3 n+2) b_{6}} \\
& A_{1}=0 \\
& A_{2}=-\frac{a q_{1}(3 n+2)}{27 n^{2} b_{3}} \\
& p=-\frac{81 n^{2}(3 n+1) b_{3}^{2}}{4 a b_{6}(3 n+2)^{2}}
\end{aligned}
$$

From Eqs. (2), (5), (19), (22) and the term $F_{1}(\xi)-F_{4}(\xi)$ (see the step 5 in Ref. [18]), we obtain the soliton solutions: 


$$
q(x, t)=\left\{\frac{(3 n+1) b_{3}}{(3 n+2) b_{6}}\left[1-\frac{4 \tanh ^{2}\left(\varepsilon \sqrt{\frac{27 n^{2}(3 n+1) b_{3}^{2}}{4 a b_{6}(3 n+2)^{2}}}(x+2 a k t)\right)}{3+\tanh ^{2}\left(\varepsilon \sqrt{\frac{27 n^{2}(3 n+1) b_{3}^{2}}{4 a b_{6}(3 n+2)^{2}}}(x+2 a k t)\right)}\right]\right\}^{\frac{1}{3 n}} e^{i(-k x+\omega t+\theta)}
$$

and

$$
q(x, t)=\left\{\frac{(3 n+1) b_{3}}{(3 n+2) b_{6}}\left[1-\frac{4 \operatorname{coth}^{2}\left(\varepsilon \sqrt{\frac{27 n^{2}(3 n+1) b_{3}^{2}}{4 a b_{6}(3 n+2)^{2}}}(x+2 a k t)\right)}{3+\operatorname{coth}^{2}\left(\varepsilon \sqrt{\frac{27 n^{2}(3 n+1) b_{3}^{2}}{4 a b_{6}(3 n+2)^{2}}}(x+2 a k t)\right)}\right)\right\}^{\frac{1}{3 n}} e^{i(-k x+\omega t+\theta)},
$$

where $b_{3} b_{6}>0$ and $a b_{6}>0$. Moreover, we obtain also the periodic solutions:

$$
q(x, t)=\left\{\frac{(3 n+1) b_{3}}{(3 n+2) b_{6}}\left[1+\frac{4 \tan ^{2}\left(\varepsilon \sqrt{-\frac{27 n^{2}(3 n+1) b_{3}^{2}}{4 a b_{6}(3 n+2)^{2}}}(x+2 a k t)\right)}{3-\tan ^{2}\left(\varepsilon \sqrt{-\frac{27 n^{2}(3 n+1) b_{3}^{2}}{4 a b_{6}(3 n+2)^{2}}}(x+2 a k t)\right)}\right]\right\}^{\frac{1}{3 n}} e^{i(-k x+\omega t+\theta)}
$$

and

$$
q(x, t)=\left\{\frac{(3 n+1) b_{3}}{(3 n+2) b_{6}}\left[1+\frac{4 \cot ^{2}\left(\varepsilon \sqrt{-\frac{27 n^{2}(3 n+1) b_{3}^{2}}{4 a b_{6}(3 n+2)^{2}}}(x+2 a k t)\right)}{3-\cot ^{2}\left(\varepsilon \sqrt{-\frac{27 n^{2}(3 n+1) b_{3}^{2}}{4 a b_{6}(3 n+2)^{2}}}(x+2 a k t)\right)}\right]\right\}^{\frac{1}{3 n}} e^{i(-k x+\omega t+\theta)}
$$

where $b_{3} b_{6}>0, a b_{6}<0$ and $\varepsilon= \pm 1$.

Type 2. Substituting $s=\frac{3 q_{1}^{2}}{16 p}$ and $r=0$ into Eqs. (21) and solving them with Maple or Mathematica, one has

$$
\begin{aligned}
& n=n, \quad \omega=-\frac{(3 n+1) b_{3}^{2}}{(3 n+2)^{2} b_{6}}-a k^{2}, \\
& A_{0}=-\frac{(3 n+1) b_{3}}{(3 n+2) b_{6}}, \\
& A_{1}=0, \\
& A_{2}=\frac{a q_{1}(3 n+2)}{9 n^{2} b_{3}}, \\
& p=-\frac{9 n^{2}(3 n+1) b_{3}^{2}}{4 a b_{6}(3 n+2)^{2}}
\end{aligned}
$$

From Eqs. (2), (5), (19), (27) and the terms $F_{5}(\xi), F_{6}(\xi)$ (see the step 5 in Ref. [18]), we arrive at the dark-soliton solutions 


$$
q(x, t)=\left\{-\frac{(3 n+1) b_{3}}{2(3 n+2) b_{6}}\left[1-\tanh \left(\varepsilon \sqrt{-\frac{9 n^{2}(3 n+1) b_{3}^{2}}{4 a b_{6}(3 n+2)^{2}}}(x+2 a k t)\right)\right]\right\}^{\frac{1}{3 n}} e^{i(-k x+\omega t+\theta)}
$$

and the singular-soliton solutions

$$
q(x, t)=\left\{-\frac{(3 n+1) b_{3}}{2(3 n+2) b_{6}}\left[1-\operatorname{coth}\left(\varepsilon \sqrt{-\frac{9 n^{2}(3 n+1) b_{3}^{2}}{4 a b_{6}(3 n+2)^{2}}}(x+2 a k t)\right)\right]\right\}^{\frac{1}{3 n}} e^{i(-k x+\omega t+\theta)},
$$

where $b_{3} b_{6}<0, a b_{6}<0$ and $\varepsilon= \pm 1$.

Type 3. Substituting $r=0$ into Eqs. (21) and solving them with Maple or Mathematica, we obtain

$$
\begin{aligned}
& n=n, \\
& \omega=-\frac{(3 n+1) b_{3}^{2}}{(3 n+2)^{2} b_{6}}-a k^{2}, \\
& A_{0}=-\frac{(3 n+1) b_{3}}{(3 n+2) b_{6}}, \quad A_{1}=0, \quad A_{2}=\frac{a q_{1}(3 n+2)}{9 n^{2} b_{3}}, . \\
& p=-\frac{9 n^{2}(3 n+1) b_{3}^{2}}{4 a b_{6}(3 n+2)^{2}}, \quad s=-\frac{a q_{1}^{2}(3 n+2)^{2} b_{6}}{12 n^{2}(3 n+1) b_{3}^{2}}
\end{aligned}
$$

From Eqs. (2), (5), (19), (30) and the term $F_{7}(\xi)-F_{10}(\xi)$ (see the step 5 in Ref. [18]), we obtain the following soliton solutions:

$$
\begin{aligned}
& q(x, t)=\left\{-\frac{(3 n+1) b_{3}}{(3 n+2) b_{6}}\left[1-\frac{2 \operatorname{sech}^{2}\left[\sqrt{-\frac{9 n^{2}(3 n+1) b_{3}^{2}}{4 a b_{6}(3 n+2)^{2}}}(x+2 a k t)\right]}{4-\left(1+\varepsilon \tanh \left[\sqrt{-\frac{9 n^{2}(3 n+1) b_{3}^{2}}{4 a b_{6}(3 n+2)^{2}}}(x+2 a k t)\right]\right)^{2}}\right]\right\}^{\frac{1}{3 n}} e^{i(-k x+\omega t+\theta)}, \\
& q(x, t)=\left\{-\frac{(3 n+1) b_{3}}{(3 n+2) b_{6}}\left[1+\frac{2 \operatorname{csch}^{2}\left[\sqrt{-\frac{9 n^{2}(3 n+1) b_{3}^{2}}{4 a b_{6}(3 n+2)^{2}}}(x+2 a k t)\right]}{4-\left(1+\varepsilon \operatorname{coth}\left[\sqrt{-\frac{9 n^{2}(3 n+1) b_{3}^{2}}{4 a b_{6}(3 n+2)^{2}}}(x+2 a k t)\right]\right)^{2}}\right]\right\}^{i(-\kappa x+\omega t+\theta)} \\
& q(x, t)=\left\{-\frac{(3 n+1) b_{3}}{(3 n+2) b_{6}}\left[1-\frac{\operatorname{sech}^{2}\left[\sqrt{-\frac{9 n^{2}(3 n+1) b_{3}^{2}}{4 a b_{6}(3 n+2)^{2}}}(x+2 a k t)\right]}{2\left(1+\varepsilon \tanh \left[\sqrt{\left.-\frac{9 n^{2}(3 n+1) b_{3}^{2}}{4 a b_{6}(3 n+2)^{2}}(x+2 a k t)\right]}\right]\right)}\right\}^{i(-k x+\omega t+\theta)}\right.
\end{aligned}
$$

and 


$$
q(x, t)=\left\{-\frac{(3 n+1) b_{3}}{(3 n+2) b_{6}}\left[1+\frac{\operatorname{csch}^{2}\left[\sqrt{-\frac{9 n^{2}(3 n+1) b_{3}^{2}}{4 a b_{6}(3 n+2)^{2}}}(x+2 a k t)\right]}{2\left(1+\varepsilon \operatorname{coth}\left[\sqrt{-\frac{9 n^{2}(3 n+1) b_{3}^{2}}{4 a b_{6}(3 n+2)^{2}}}(x+2 a k t)\right]\right)}\right]\right\}^{\frac{1}{3 n}} e^{i(-\kappa x+\omega t+\theta)}
$$

provided that $b_{3} b_{6}<0, a b_{6}<0$ and $\varepsilon= \pm 1$.

\section{Addendum to the Kudryashov's method}

Recently, Kudryashov has suggested the approach to integrating equations of the type which is studied in the present work [9]. We summarize our addendum to this Kudryashov's method as follows.

First, we assume that Eq. (8) has the formal solution

$$
H(\xi)=\sum_{S=0}^{M} B_{S} R^{S}(\xi)
$$

where $B_{S}(S=0,1,2, \ldots, M)$ are constants to be determined $\left(B_{M} \neq 0\right)$ and $R(\xi)$ satisfies the auxiliary ordinary differential equation

$$
{R^{\prime}}^{2}(\xi)=R^{2}(\xi)\left[1-\chi R^{2 p}(\xi)\right] \ln ^{2} k, 0<k \neq 1,
$$

with $\chi$ being a constant. It is easy to show that Eq. (36) has the solutions

$$
R(\xi)=\left[\frac{4 A}{4 A^{2} k^{p \xi}+\chi k^{-p \xi}}\right]^{\frac{1}{p}}
$$

where $A$ is a nonzero constant and $p$ a positive integer. In order to apply the method [6, 19], we first balance $H H^{\prime \prime}$ with $H^{4}$ in Eq. (8), thus arriving at

$$
2 M+2 p=4 M \Rightarrow M=p .
$$

Let us now discuss the following specific cases.

Case 1. Let us choose $p=2$ and $M=2$. Then we deduce from Eq. (35) the relation

$$
H(\xi)=B_{0}+B_{1} R(\xi)+B_{2} R^{2}(\xi),
$$

where $B_{0}, B_{1}$ and $B_{2}$ are constants to be determined $\left(B_{2} \neq 0\right)$ and the function $R(\xi)$ satisfies Eq. (36). Substituting Eq. (39) and (36) into Eq. (8), collecting all the coefficients near each power of $[R(\xi)]^{q_{1}}$ and $\left[R^{\prime}(\xi)\right]^{q_{2}},\left(q_{1}=0,1,2, \ldots 8, q_{2}=0,1\right)$ and setting each of these coefficients to zero, we obtain a system of algebraic equations, which can be solved using Maple. We have

$$
\begin{aligned}
& n=n, \omega=\frac{4 a \ln ^{2} k}{9 n^{2}}-a k^{2}, \\
& B_{0}=B_{1}=0, \quad B_{2}=\sqrt{\frac{4 a \chi(3 n+1) \ln ^{2} k}{9 n^{2} b_{6}},} \\
& b_{3}=0,
\end{aligned}
$$

where $a \chi b_{6}>0$. In this case we conclude that Eq. (1) has the soliton solutions 


$$
q(x, t)=\left\{\sqrt{\frac{4 a \chi(3 n+1) \ln ^{2} k}{9 n^{2} b_{6}}}\left[\frac{4 A}{4 A^{2} k^{2(x+2 a k t)}+\chi k^{-2(x+2 a k t)}}\right]\right\}^{\frac{1}{3 n}} e^{i(-k x+\omega t+\theta)}
$$

In particular, if we have $\chi=4 A^{2}$ in Eq. (41), then Eq. (1) has the bright-soliton solutions

$$
q(x, t)=\left\{\sqrt{\frac{4 a(3 n+1) \ln ^{2} k}{9 n^{2} b_{6}}} \operatorname{sech}[2(x+2 a k t) \ln k]\right\}^{\frac{1}{3 n}} e^{i(-k x+\omega t+\theta)},
$$

provided that $a b_{6}>0$. On the other hand, if we set $\chi=-4 A^{2}$ in Eq. (41), Eq. (1) has the singularsoliton solutions

$$
q(x, t)=\left\{\sqrt{-\frac{4 a(3 n+1) \ln ^{2} k}{9 n^{2} b_{6}}} \operatorname{csch}[2(x+2 a k t) \ln k]\right\}^{\frac{1}{3 n}} e^{i(-k x+\omega t+\theta)},
$$

provided that $a b_{6}<0$.

Case 2. Let us choose $p=3$ and $M=3$. We obtain from Eq. (35) that

$$
H(\xi)=B_{0}+B_{1} R(\xi)+B_{2} R^{2}(\xi)+B_{3} R^{3}(\xi),
$$

where $B_{0}, B_{1}, B_{2}$ and $B_{3}$ are constants to be determined $\left(B_{3} \neq 0\right)$ and the function $R(\xi)$ satisfies Eq. (36). Substituting Eqs. (44) and (36) into Eq. (8), collecting all the coefficients near each power of $[R(\xi)]^{q_{1}}$ and $\left[R^{\prime}(\xi)\right]^{q_{2}} \quad\left(q_{1}=0,1,2, \ldots 12, q_{2}=0,1\right)$ and setting each of these coefficients to zero, we obtain a system of algebraic equations which can be solved using Maple. As a result, we have

$$
\begin{aligned}
& n=n, \omega=\frac{a \ln ^{2} k}{n^{2}}-a k^{2}, \\
& B_{0}=B_{1}=B_{2}=0, B_{3}=\sqrt{\frac{a \chi(3 n+1) \ln ^{2} k}{n^{2} b_{6}},} \\
& b_{3}=0,
\end{aligned}
$$

provided that $a \chi b_{6}>0$. In this case, Eq. (1) has the soliton solutions:

$$
q(x, t)=\left\{\sqrt{\frac{a \chi(3 n+1) \ln ^{2} k}{n^{2} b_{6}}}\left[\frac{4 A}{4 A^{2} k^{3(x+2 a k t)}+\chi k^{-3(x+2 a k t)}}\right]\right\}^{\frac{1}{3 n}} e^{i(-k x+\omega t+\theta)} .
$$

In particular, if we set $\chi=4 A^{2}$ in Eq. (46), Eq. (1) has the bright-soliton solutions:

$$
q(x, t)=\left\{\sqrt{\frac{4 a(3 n+1) \ln ^{2} k}{n^{2} b_{6}}} \operatorname{sech}[3(x+2 a k t) \ln k]\right\}^{\frac{1}{3 n}} e^{i(-k x+\omega t+\theta)},
$$

provided that $a b_{6}>0$. Finally, if we set $\chi=-4 A^{2}$ in Eq. (46), Eq. (1) has the singular-soliton solutions:

$$
q(x, t)=\left\{\sqrt{-\frac{4 a(3 n+1) \ln ^{2} k}{n^{2} b_{6}}} \operatorname{csch}[3(x+2 a k t) \ln k]\right\}^{\frac{1}{3 n}} e^{i(-k x+\omega t+\theta)}
$$


provided that $a b_{6}<0$. Similarly, one can find many other solutions by choosing the other values of $p$ and $M$. Note that the case $p=1$ and $M=1$ has been omitted since it does not work.

\section{Conservation laws}

From Eq. (7), one can see that Eq. (1) collapses into the following form for integrability purposes:

$$
i q_{t}+a q_{x x}+\left(b_{3}|q|^{3 n}+b_{6}|q|^{6 n}\right) q=0 .
$$

Its bright single-soliton solutions are given by

$$
q(x, t)=A \operatorname{sech}^{\frac{1}{3 n}}[B(x-v t)] e^{i(-k x+\omega t+\theta)},
$$

where $A$ is the amplitude of the soliton, $B$ its inverse width and $v$ its velocity. The model given by Eq. (49) reveals three conserved quantities as indicated earlier in Ref. [2]: the power $P$, the linear momentum $M$ and the Hamiltonian $H$ [19]. They are given by (see Ref. [20])

$$
\begin{aligned}
& P=\int_{-\infty}^{\infty}|q|^{2} d x=\frac{A^{2}}{B} \frac{\Gamma\left(\frac{1}{6 n}\right) \Gamma\left(\frac{1}{2}\right)}{\Gamma\left(\frac{1}{6 n}+\frac{1}{2}\right)}, \\
& M=\int_{-\infty}^{\infty}\left(q^{*} q_{x}-q q_{x}^{*}\right) d x=\frac{a k A^{2}}{B} \frac{\Gamma\left(\frac{1}{6 n}\right) \Gamma\left(\frac{1}{2}\right)}{\Gamma\left(\frac{1}{6 n}+\frac{1}{2}\right)}
\end{aligned}
$$

and

$$
\begin{aligned}
& H=\int_{-\infty}^{\infty}\left(a\left|q_{x}\right|^{2}-\frac{2 b_{3}}{n+2}|q|^{3 n+2}-\frac{b_{6}}{3 n+1}|q|^{2(3 n+1)}\right) d x \\
& =\frac{a A^{2}}{3 n(3 n+2) B}\left\{3 n(3 n+2) k^{2}+B^{2}\right\} \frac{\Gamma\left(\frac{1}{3 n}\right) \Gamma\left(\frac{1}{2}\right)}{\Gamma\left(\frac{1}{3 n}+\frac{1}{2}\right)}-\frac{6 n b_{3} A^{3 n+2}}{(3 n+2) B} \frac{\Gamma\left(\frac{1}{3 n}+\frac{1}{2}\right) \Gamma\left(\frac{1}{2}\right)}{\Gamma\left(\frac{1}{3 n}\right)} \\
& -\frac{2 b_{6} A^{2(3 n+1)}}{(3 n+1)(3 n+2) B} \frac{\Gamma\left(\frac{1}{3 n}\right) \Gamma\left(\frac{1}{2}\right)}{\Gamma\left(\frac{1}{3 n}+\frac{1}{2}\right)} .
\end{aligned}
$$

\section{Conclusion}

The main aim of the present work has been to find new exact solutions of the problem linked with the Kudryashov's sextic power law for the nonlinear refractive index. These solutions include the ones associated with the optical solitons. Three different integration algorithms have been utilized to solve the problem: the unified Riccati equation, the new mapping method, and the technique which represents our addendum to the earlier N. A. Kudryashov's method.

After extensive mathematics implemented with the three integration algorithms, we have arrived at the same conclusion: the N. A. Kudryashov's model with the sextic power-law nonlinearity collapses to the special case of triple power-law format, as given by Eq. (49) $[2,3,5$, 19]. If one hypothetically replaces $3 n$ with $m$ in Eq. (49), this picture becomes very clear [2, 3, 5, 19]. Hence, the final results of our study prove the claims declared by us. 


\section{Acknowledgements}

The research work of one of us (M. R. B.) was supported by the grant NPRP 11S-1126-170033 from QNRF

\section{References}

1. Biswas A, 2020. Optical soliton cooling with polynomial law of nonlinear refractive index. J. Opt. 49: 580-583.

2. Biswas A, Ekici M, Zhou Q, Alzahrani A K and Belic M, 2020. Conservation laws for optical solitons with polynomial and triple-power laws of refractive index. Optik. 202: 163476.

3. Jawad A J M, Abu-Al Shaeer M J, Zayed E M E, Alngar M E M, Biswas A, Ekici M, Alzahrani A K and Belic M R, 2020. Optical soliton perturbation with exotic forms of nonlinear refractive index. Optik. 223: 165329.

4. Kaur G, Kaur G and Sharma S, 2020. New dispersion-compensated Raman-amplifier cascade with a single-pump parametric amplifier for dense wavelength-division multiplexing. Ukr. J. Phys. Opt. 21: 35-46.

5. Kohl R W, Biswas A, Zhou Q, Ekici M, Alzahrani A K and Belic M R, 2020. Optical soliton perturbation with polynomial and triple-power laws of refractive index by semi-inverse variational principle. Chaos Solit. Fract. 135: 109765.

6. Kudryashov N A, 2019. First integrals and general solution of the traveling wave reduction for Schrödinger equation with anti-cubic nonlinearity. Optik. 185: 665-671.

7. Kudryashov N A, 2019. General solution of traveling wave reduction for the KunduMukherjee-Naskar model. Optik. 186: 22-27.

8. Kudryashov N A, 2019. First integrals and general solution of the Fokas-Lenells equation. Optik. 195: 163135.

9. Kudryashov N A, 2020. Mathematical model of propagation pulse in optical fiber with power nonlinearities. Optik. 212: 164750.

10. Kudryashov N A, 2019. A generalized model for description of propagation pulses in optical fiber. Optik. 189: 42-52.

11. Kudryashov N A and Antonova E V, 2020. Solitary waves of equation for propagation pulse with power nonlinearities. Optik. 217: 164881.

12. Kudryashov N A, 2020. Method for finding highly dispersive optical solitons of nonlinear differential equations. Optik. 206: 163550.

13. Kudryashov N A, 2020. Highly dispersive optical solitons of equation with various polynomial nonlinearity law. Chaos Solit. Fract. 140: 110202.

14. Kudryashov N A, 2020. Periodic and solitary waves in optical fiber Bragg gratings with dispersive reflectivity. Chin. J. Phys. 66: 401-405.

15. Kudryashov N A, 2020. First integrals and general solution of the complex Ginzburg-Landau equation. Appl. Math. Comput. 386: 125407.

16. Kudryashov N A, 2020. Optical solitons of the model with arbitrary refractive index. Optik. 224: 165391.

17. Sirendaoreji N, 2017. Unified Riccati equation expansion method and its application to two new classes of Benjamin-Bona-Mahony equations. Nonlin. Dyn. 89: 333-344.

18. Zeng $\mathrm{X}$ and Yong $\mathrm{X}, 2008$. A new mapping method and its applications to nonlinear partial differential equations. Phys. Lett. A. 372: 6602-6607. 
19. Zayed E M E, Alngar M E M, Biswas A, Kara A H, Moraru L, Ekici M, Alzahrani A K and Belic M R, 2020. Solitons and conservation laws in magneto-optic waveguides with triplepower law nonlinearity. J. Opt. 49: 584-590.

20. Zhidkov P E. Korteweg-de Vries and nonlinear Schrödinger equations: qualitative theory. Springer Verlag: New York (2001).

Elsayed M. E. Zayed, Reham M. A. Shohib, Mohamed E. M. Alngar, Anjan Biswas, Mehmet Ekici, Salam Khan, Abdullah Khamis Alzahrani and Milivoj R. Belic. 2021 Optical solitons and conservation laws associated with Kudryashov's sextic power-law nonlinearity of refractive index. Ukr.J.Phys.Opt. 22: 38 - 49. doi: 10.3116/16091833/22/1/38/2021

Анотація. Одержано розв'язки у вигляді «яскравих», «темних» $i$ сингулярних оптичних солітонів для ефекту самофазної модуляиії, який належить до типу степеневої нелінійності показника заломлення шостого порядку, щяо була раніше описана Н. А. Кудряшовим. Впроваджено три різні схеми інтегрування. Це уніфіковане рівняння Ріккаті, наш новий метод відображення та розвиток загальноприйнятого методу Н. А. Кудрямова. Наведено вирази для всіх солітонів та перераховано критерії їхнього існування. Одержано також три відповідні закони збереження. 